\title{
The prognostic value of fat invasion and tumor expansion in the hilar veins in $\mathrm{pT} 3 \mathrm{a}$ renal cell carcinoma
}

\author{
Viktoria Stühler $^{1} \cdot$ Steffen Rausch $^{1} \cdot$ Katharina Kroll $^{1} \cdot$ Marcus Scharpf $^{2} \cdot$ Arnulf Stenzl $^{1} \cdot$ Jens Bedke ${ }^{1}$ (i)
}

Received: 9 November 2020 / Accepted: 10 February 2021 / Published online: 27 February 2021

(c) The Author(s) 2021

\begin{abstract}
Purpose The 7th TNM classification summarizes renal cell carcinoma (RCC) with perirenal (PFI) and/or sinus fat invasion (SFI) as well as hilar vein involvement (RVI) as pT3a tumors. In this study, we aimed to determine the prognostic value of fat invasion (FI) in the different compartments and RVI for medium-term cancer-specific-survival (CSS) in pT3a RCC. Materials and methods Patients with pT3a RCC were identified using an institutional database. All original pathological reports were reclassified according to the 7th TNM edition. The prognostic value of FI as well as divided into PFI, SFI, combined PFI + SFI, and RVI for CSS was assessed using univariate and multivariate Cox-regression analysis. Survival was estimated using the Kaplan-Meier method.

Results Median follow-up in 184 pT3a tumors was 38 months. FI was detectable in 153 patients (32.7\% PFI, 45.1\% SFI, 22.2\% PFI + SFI), 31 patients showed RVI alone. Combined PFI + SFI increased the risk of cancer-related death compared to PFI (HR 3.11, $p<0.01$ ), SFI (HR 1.84, $p=0.023$ ) or sole RVI (HR 2.12, $p=0.025$ ). In multivariate analysis, a combined PFI + SFI vs. PFI or SFI as the only compartment involved was confirmed as independent prognostic factor (HR 1.83, $p=0.029)$. Patients with FI and simultaneous RVI had significantly shorter CSS (HR 2.63, $p<0.01$ ). In an unweighted model, the difference between patients with combined PFI+SFI and RVI and those with PFI alone was highest (HR 4.01, $p=0.029)$. Conclusions These results underline the subdivision of pT3a RCC depending on the location of FI and RVI for patient stratification.
\end{abstract}

Keywords Fat invasion $\cdot$ Hilar vein involvement $\cdot$ pT3a stage $\cdot$ Renal cell carcinoma

$\begin{array}{ll}\text { Abbreviations } \\ \text { CI } & \text { Confidence interval } \\ \text { CSS } & \text { Cancer specific survival } \\ \text { FI } & \text { Fat invasion } \\ \text { G } & \text { Grading } \\ \text { PFI } & \text { Perirenal fat invasion } \\ \text { HR } & \text { Hazard ratio } \\ \text { L } & \text { Invasion into lymph vessels } \\ \text { M } & \text { Distant metastasis } \\ \text { N } & \text { Regional lymph nodes } \\ \text { R } & \text { Resection status } \\ \text { RCC } & \text { Renal cell carcinoma } \\ \text { RVI } & \text { Hilar vein involvement }\end{array}$

Jens Bedke

bedke@live.com

1 Department of Urology, University of Tuebingen, Tübingen, Germany

2 Department of Pathology, University of Tuebingen, Tübingen, Germany

$\begin{array}{ll}\text { SFI } & \text { Sinus fat invasion } \\ \text { T } & \text { Primary tumor } \\ \text { TNM } & \text { Tumor-node-metastasis classification system } \\ \text { V } & \text { Invasion into veins }\end{array}$

\section{Introduction}

Overall survival of patients with renal cell carcinoma (RCC) varies widely. The likelihood for patients with localized RCC treated in curative intention with tumor resection to suffer relapse with lymph node or distant metastases is up to $30 \%$ [1]. After tumor resection, an accurate assessment of the risk of relapse is important to offer a risk-adapted followup frequency and, if available, the possibility of adjuvant therapy, taking into account the outstanding study results with immune checkpoint inhibitors. Tumor invasion in the renal fat is often divided into perirenal (PFI) and perihilar (SFI) fat invasion and is detected in $5.1-18.5 \%$ of cases [2, 3]. The 2010 TNM classification led to a major change in 
RCC classification. Up to this time, tumors with invasion of the perinephric tissue or the adrenal gland were classified as pT3a tumors, and those with expansion into the hilar veins, their segmental branches or the vena cava below the diaphragm as pT3b tumors. Due to the revision of the TNM classification in 2010, tumors with FI and even extension into the hilar veins or their segmental (muscle-containing) branches are now summarized as pT3a tumors. In the new classification, a pT3b tumor is characterized by a tumor expansion into the vena cava below the diaphragm [4]. The changes in TNM classification are summarized in Fig. 1 and illustrated with exemplary histologic images. However, the prognostic value of FI with subdivision in the different fat compartments PFI and SFI with or without hilar vein involvement (RVI) is controversially discussed.

In this study, we examined the prognostic value of FI, divided in PFI, SFI, and combined PFI + SFI, and RVI for medium-term cancer-specific survival (CSS) in patients with pT3a RCC to improve risk stratification. These results shall help to adjust the follow-up frequency after kidney surgery, and to identify patients at high risk of recurrence who may benefit from adjuvant treatment strategies.

\section{Patients and methods}

A flowchart showing the process of patient selection for this retrospective study from a total cohort with 754 patients after radical or partial nephrectomy for RCC between 1993 and 2003 at the Department of Urology, University of Tuebingen, Germany, is deposited in Fig. 2. Primary histopathologic analysis was carried out by uro-pathologists, whereas re-assessment according to 7th TNM edition from 2010 was performed by the review of original pathological reports by two authors (V.S. and J.B.). In case of discrepancy, reports were re-evaluated by the uro-pathologist (M.S.). Finally, 184 patients with histological diagnosed pT3a RCC were included in the study. Data were collected in an institutional database and included documentation of sex, age, height, weight, nicotine or alcohol use, time of death or time of last follow-up, and cause of death (RCC-related or RCC-independent). The following tumor-specific parameters were also collected: fat invasion (PFI, SFI, or combined PFI+SFI), histological subtype, tumor size, TNM classification according to UICC 2010, grading, L-, V-, and R-stages, the presence of necrosis and/or sarcomatoid parts and capsular or adrenal invasion. Because fat invasion (FI) correlated with $\mathrm{N}$ - and $\mathrm{M}$-stages in preliminary analyses (data not shown), cM1 patients were also included in first analyses. The last follow-up was carried out in January 2020. The study was approved by the local ethics committee (078/2012/B02).

The primary aim of this study was the retrospective evaluation of the prognostic value of FI into the different renal compartments in correlation with or without RVI for medium-term CSS in pT3a RCC. All patients underwent surgical resection of the tumors renal mass according to institional surgical guidelines, in which nephron sparing surgery is recommend compared to radical nephrectomy if technical feasible either by open or laparoscopic approach.

\section{Statistical analysis}

In a first step, we performed a descriptive statistic of the clinicopathological parameters. The prognostic influence of FI as well as divided into the different fat compartments or a sole RVI on CSS was investigated using univariate and multivariate Cox-regression analysis. Factors that showed significance in univariate analysis were evaluated using multivariate models. Survival analyses were estimated using the Kaplan-Meier method and significant differences were determined using the log-rank test for univariate analysis. All statistical analyses were performed with SPSS version 26 (released 2019, Armonk, NY: IBM Corp) and Windows Microsoft Excel (Office 12). Statistical significance was defined as $p<0.05$.

\section{Results}

This study involved 184 consecutive pT3a RCC patients. Due to the re-classification according to the 7th TNM edition from 2010, 60 originally classified pT3b tumors were reclassified as pT3a tumors. Patient characteristics are given in Fig. 1. The results of univariate analysis of clinicopathological parameters showing significant differences in CSS, such as tumor diameter, grading, L-, V-, and R-stages, tumor necrosis, sarcomatoid differentiation, capsular infiltration, $\mathrm{N}$ - and M-stages are listed in Table 1A. Because of a strong imbalance ( $n=172$ for clear cell RCC and $n=12$ for nonclear cell RCC), histological subtype was not included in the univariate and multivariate Cox-regression models. Kaplan-Meier analyses of the 184 patients are shown in Fig. 2 and for the univariate Cox-regression analyses in Table 1. Classified according to the location of FI, PFI was present in 50 (32.7\%), SFI in $69(45.1 \%)$ and a combined PFI + SFI in $34(22.2 \%)$ patients, respectively. 31 (16.8\%) patients showed no FI, however, were classified as pT3a tumors due to RVI. Median follow-up was 32 months (0-228 months) with 85.3\% follow-up rate in January 2020. During the follow-up period, 109 (59.2\%) patients died, with 79 (42.9\%) patients of cancer-specific death.

The estimated 2- and 5-year CSS rates of the whole collective depending on FI and divided in the different fat compartments are summarized in Table 1A. For the whole collective, FI itself was not a significant parameter for CSS compared to patients with only RVI (HR 1.18, $p=0.591$, 


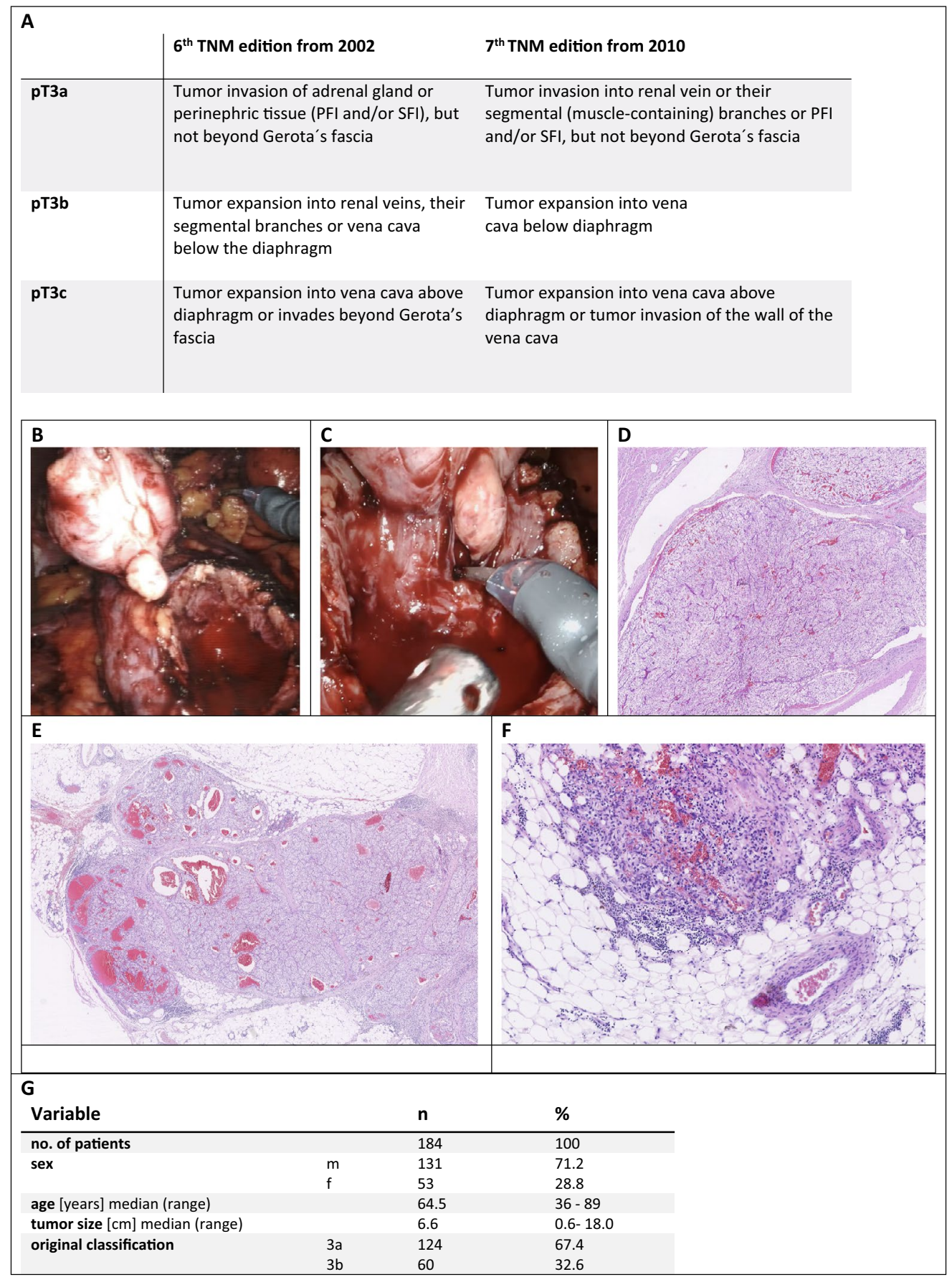

Fig. 1 a CSS of the total collective (median 94 months). b CSS of patients with FI depending on RVI, $p<0.001$. $\mathbf{c}$ CSS depending on affected area of FI in the total population; SFI vs. PFI: $p=0.079$, PFI vs. PFI +SFI: $p<0.001$, SFI vs. PFI + SFI: $p=0.023$; no FI only RVI vs. PFI + SFI: $p=0.025$. d CSS depending on affected area of FI in the M0 subcollective; SFI vs. PFI: $p=0.667$, PFI vs. PFI + SFI: $p=0.226$, SFI vs. PFI + SFI: $p=0.408$; no FI only RVI vs. PFI + SFI: $p=0.559$. All $p$ values for the overall comparison (log-rank test); significance in pairwise comparison. e Unweighted prognostic risk stratification model 1: CSS of patients depending on the variables M-stage, L-stage, and RVI; M0 L1 RVI1 vs. M0 L0 RVI0: $p<0.001$. f Unweighted prognostic risk stratification model 2: CSS of patients depending on the variable M-stage, PFI, SFI, and combined PFI and SFI with or without RVI; PFI+RVI M0 vs. PFI without RVI M0: $p=0.043$; SFI +RVI M0 vs. SFI without RVI M0: $p=0.409$; $\mathrm{PFI}+\mathrm{SFI}+\mathrm{RVI}$ M0 vs. PFI + SFI without RVI M0: $p=0.202$. g Tabular summary of the models 1 and 2 . h Flowchart showing the process of patient selection for this retrospective study, starting from the total cohort of patients after radical or partial nephrectomy for RCC between 1993 and 2003 at the Department of Urology, University of Tuebingen. $F I$ fat invasion, $L$ invasion into lymph vessels, $M$ distant metastasis, PFI perirenal fat invasion, $R V I$ renal vein involvement, $S F I$ hilar fat invasion 


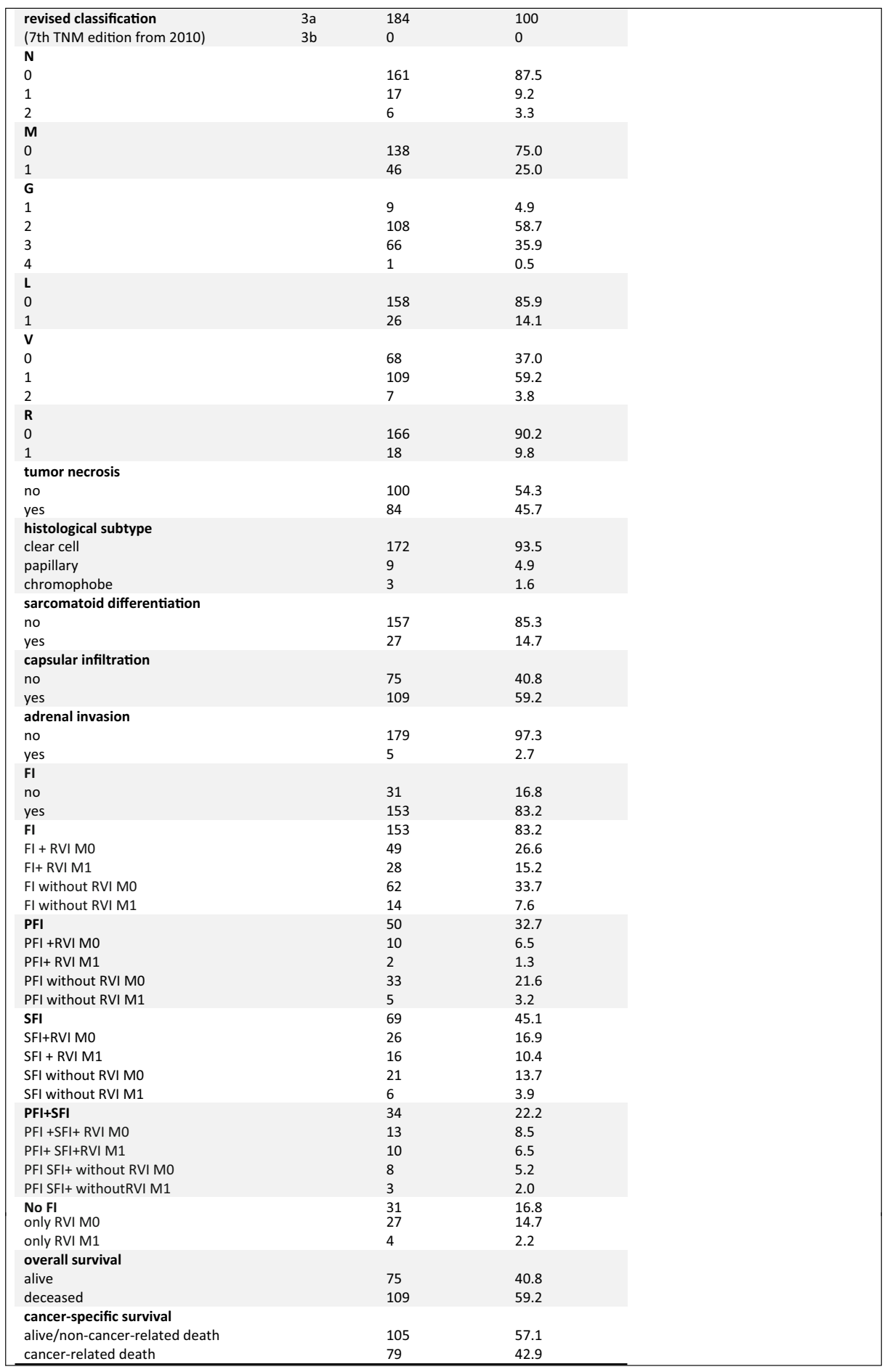

Fig. 1 (continued)

see Table 1A). Patients with FI and simultaneous RVI had a significantly shorter CSS compared to patients with sole FI (median CSS 42 months vs. not reached, HR 2.63, $p<0.01$ ). Further, there was a significant difference between the subgroup of patients with combined PFI + SFI and patients with no FI but RVI (32 vs. 97 months, HR 2.12, $p=0.025$ ). The difference in CSS was not significant between patients with SFI or PFI $(p=0.079)$. In univariate analysis, a combined PFI + SFI significantly increased the risk of cancer-related death compared to PFI (HR 3.11, $p<0.01$ ) or SFI (HR 1.84, $p=0.023$ ), see Table 1B. The 2- and 5-year CSS rates for the subgroup of patients without distant metastases (M0, 


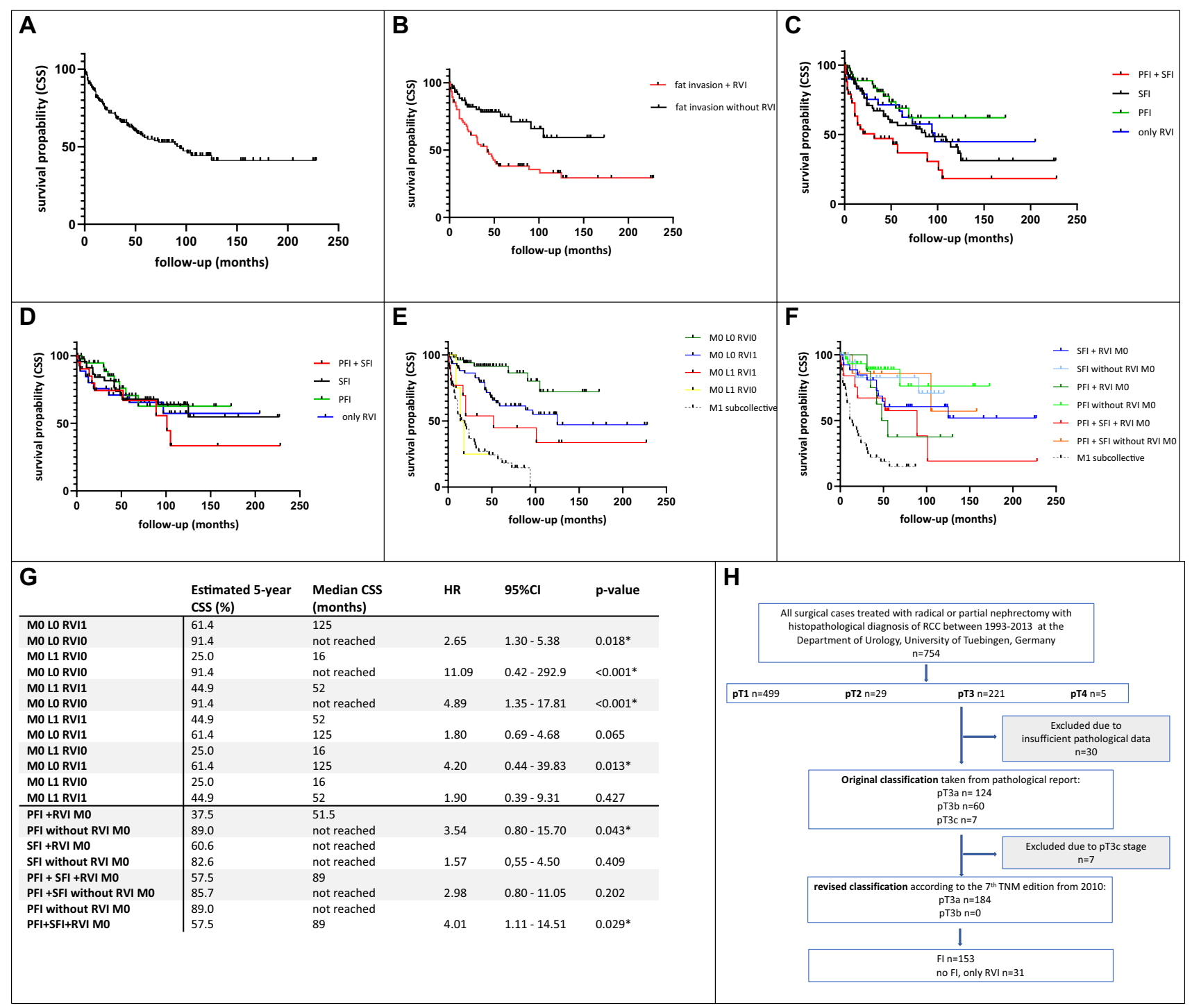

Fig. 2 a 6th TNM edition from 2002 and 7th TNM edition from 2010 for pT3 subclassification of RCC. Below, two exemplary intraoperative images of RCC with invasion into the segmental (muscle-containing) branches of the hilar veins are shown in the top row $(\mathbf{b}, \mathbf{c})$ with the matching histologic image (d). Below that, a histologic overview image of an RCC with perirenal fat invasion is shown with cor-

$n=138$ ) as well as the univariate analysis of the various fat compartments showed no significant difference in CSS and are summarized in Table 1A, B. Data of the M1 subcollective $(n=46)$ should be interpreted with caution due to the small number of patients and are also given in Table 1B.

In the multivariate analyses, the parameters M-stage, tumor diameter stratified by the median, tumor grading ( $\geq 3 / 4)$, L-stage, tumor necrosis, sarcomatoid differentiation and RVI were adjusted in addition to FI and substratified in the various fat compartments. As shown in model 1 in Table 1C, FI was no independent prognostic factor for CSS (HR 1.24, $p=0.523$ ). On the other hand, the values responding magnification on the right side $(\mathbf{e}, \mathbf{f})$. $\mathbf{g}$ Detailed patient characteristics of the investigated pT3a RCC cohort. FI fat invasion, $G$ grading, $L$ invasion into lymph vessels, $M$ distant metastasis, $N$ regional lymph nodes, $P F I$ perirenal fat invasion, $R$ resection status, $R V I$ renal vein involvement, $S F I$ hilar fat invasion, $T$ primary tumor, $V$ invasion into veins

of M- and L-stages and RVI were identified as independent prognostic parameters for CSS. When considering the affected FI compartments in multivariate analyses (model 2-5 in Table 1D), combined PFI + SFI compared to PFI or SFI as single affected compartment was an independent prognostic factor for poorer tumor-dependent survival (HR $1.83, p=0.029$, see model 5). However, models 3 and 4 in Table 1D show that the presence of a combined PFI + SFI compared to an isolated PFI (HR 1.75, $p=0.172$ ) or SFI (HR 1.75, $p=0.062$ ) could not be identified as independent prognostic factors for CSS. However, RVI was identified as an independent prognostic factor for CSS in models 1,3 , and 
Table 1 A Estimated 2- and 5-year CSS, median CSS and univariate Cox-regression analysis of clinicopathological parameters, B univariate Cox-regression analysis of the different fat compartements, C model 1 (CSS) - multivariate analyses of clinical and pathological parameters with regard to FI, $n=18$, D: model 2 (CSS, SFI vs.
PFI), model 3 (CSS, PFI + SFI vs. PFI), model 4 (CSS, PFI + SFI vs. $\mathrm{SFI}$ ), and model 5 (CSS, combined PFI + SFI vs. PFI or SFI as sole involved compartment)-multivariate analyses of clinical and pathological factors of the total collective

\begin{tabular}{|c|c|c|c|c|c|c|}
\hline \multicolumn{7}{|l|}{ A } \\
\hline & \multirow{2}{*}{$\begin{array}{l}\text { Estimated 2-year } \\
\text { CSS }(\%)\end{array}$} & \multirow{2}{*}{$\begin{array}{l}\text { Estimated 5-year } \\
\text { CSS }(\%)\end{array}$} & \multirow[t]{2}{*}{ Median CSS (months) } & \multicolumn{3}{|c|}{ Univariate Cox-regression analysis } \\
\hline & & & & HR & $95 \% \mathrm{CI}$ & $p$ value \\
\hline Whole collective & 71.2 & 55.8 & 94.00 & & & \\
\hline FI & 70.9 & 53.5 & 91.0 & & & \\
\hline No FI, only RVI & 75.3 & 66.9 & 97.0 & 1.18 & $0.67-2.07$ & 0.591 \\
\hline FI with RVI & 61.0 & 38.1 & 42.0 & & & \\
\hline FI without RVI & 82.1 & 75.0 & Not reached & 2.63 & $1.62-4.26$ & $<0.001 *$ \\
\hline \multicolumn{7}{|l|}{ MO subcollective } \\
\hline FI & 86.1 & 70.0 & Not reached & & & \\
\hline No FI, only RVI & 75.7 & 65.5 & Not reached & 0.87 & $0.40-1.87$ & 0.397 \\
\hline \multicolumn{7}{|l|}{ M1 subcollective } \\
\hline FI & 33.0 & 15.1 & 14 & & & \\
\hline No FI, only RVI & 75.0 & 50.0 & 67.5 & 1.99 & $0.88-4.49$ & 0.139 \\
\hline PFI & 88.8 & 70.00 & Not reached & & & \\
\hline SFI & 70.9 & 56.5 & 87.0 & \multicolumn{3}{|c|}{ For more details see Table 1B } \\
\hline $\mathrm{PFI}+\mathrm{SFI}$ & 50.6 & 36.8 & 32.0 & & & \\
\hline \multicolumn{7}{|l|}{ Tumor diameter } \\
\hline$<$ Median & 79.8 & 67.0 & Not reached & & & \\
\hline$\geq$ Median & 62.5 & 43.4 & 51 & 0.45 & $0.29-0.71$ & $<0.001^{*}$ \\
\hline \multicolumn{7}{|l|}{$N$} \\
\hline No & 76.0 & 61.5 & 125 & & & \\
\hline $\mathrm{N} 1 / \mathrm{N} 2$ & 43.5 & 21.7 & 17 & 0.30 & $0.14-0.64$ & $<0.001^{*}$ \\
\hline \multicolumn{7}{|l|}{$M$} \\
\hline M0 & 84.0 & 68.3 & Not reached & & & \\
\hline M1 & 36.8 & 21.3 & 19 & 0.22 & $0.12-0.40$ & $<0.001^{*}$ \\
\hline \multicolumn{7}{|l|}{ Grading } \\
\hline $\mathrm{G} 1 / \mathrm{G} 2$ & 81.1 & 65.6 & Not reached & & & \\
\hline G3/G4 & 55.4 & 38.8 & 35 & 0.43 & $0.26-0.69$ & $<0.001^{*}$ \\
\hline \multicolumn{7}{|l|}{$V$} \\
\hline V0 & 83.3 & 77.7 & Not reached & & & \\
\hline $\mathrm{V} 1 / \mathrm{V} 2$ & 65.3 & 45.6 & 51 & 0.36 & $0.23-0.56$ & $<0.001^{*}$ \\
\hline \multicolumn{7}{|l|}{$L$} \\
\hline L0 & 78.3 & 61.2 & 105 & & & \\
\hline L1 & 34.6 & 25.2 & 15.5 & 0.34 & $0.17-0.70$ & $<0.001^{*}$ \\
\hline \multicolumn{7}{|l|}{ Tumor necrosis } \\
\hline No & 83.6 & 63.8 & 125 & & & \\
\hline Yes & 56.9 & 45.7 & 44 & 0.48 & $0.30-0.75$ & $<0.001^{*}$ \\
\hline \multicolumn{7}{|c|}{ Sarcomatoid differentiation } \\
\hline No & 75.0 & 60.0 & 125 & & & \\
\hline Yes & 53.0 & 31.9 & 31 & 0.42 & $0.21-0.86$ & $<0.001^{*}$ \\
\hline \multicolumn{7}{|c|}{ Capsular infiltration } \\
\hline No & 81.4 & 61.7 & 125 & & & \\
\hline Yes & 64.7 & 51.5 & 62 & 0.64 & $0.41-0.99$ & 0.050 \\
\hline \multicolumn{7}{|l|}{$R$} \\
\hline 0 & 74.3 & 58.5 & 97 & & & \\
\hline $1 / 2$ & 47.1 & 32.3 & 20 & 0.53 & $0.24-1.20$ & $0.047 *$ \\
\hline
\end{tabular}


Table 1 (continued)

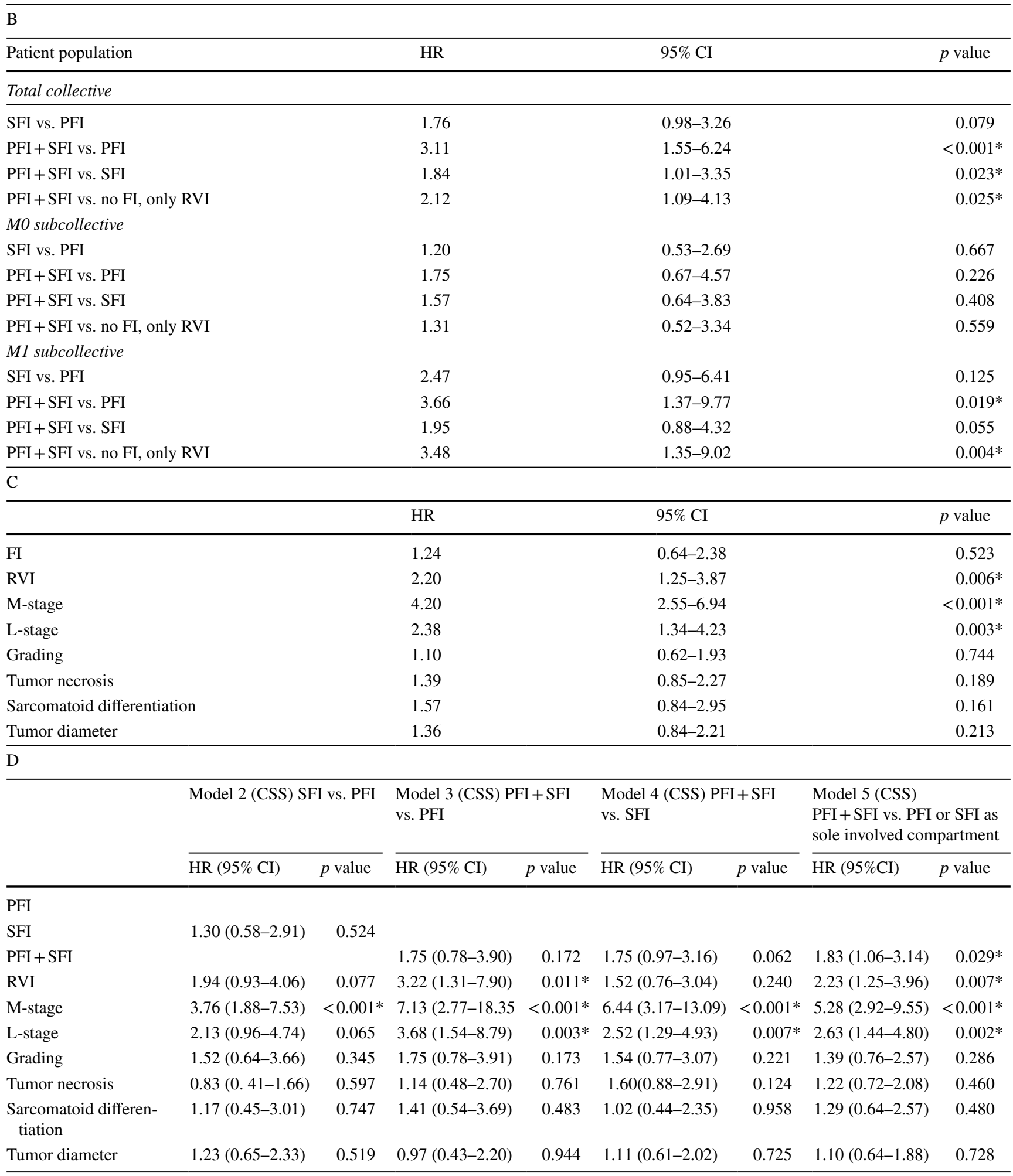

$F I$ fat invasion, $G$ grading, $L$ invasion into lymph vessels, $M$ distant metastasis, $N$ regional lymph nodes, $P F I$ perirenal fat invasion, $R$ resection status, $R V I$ renal vein involvement, $S F I$ hilar fat invasion, $V$ invasion into veins

*Statistically significant $(p<0.05)$ 
5. Further, the presence of distant metastases and L-stage were identified as independent predictive factors for CSS in all multivariate Cox-regression models.

Finally, we created an unweighted prognostic risk stratification model for Kaplan-Meier analysis to further investigate the independent prognostic parameters M-stage, L-stage, and RVI, see Fig. 2e, g. This model stratified pT3a M0 patients into groups that differ significantly in their CSS. The subgroup of patients with FI M0 L0 RVI0 showed a significantly improved CSS compared to those with FI M0 L1 RVI1 (5-year CSS rate $91.4 \%$ vs. $44.9 \%$, HR $4.89, p<0.01$ ). In our study, patients of the group M0 L1 RVI0 showed an even worse median CSS of 16 months. However, due to the small number of patients in this subgroup $(n=4)$, this result should be assessed with caution. In a second risk model, we were able to demonstrate that simultaneous RVI reduced CSS in M0 patients, seen for all affected fat compartments. This difference was significant for patients with PFI alone showing a 5-year CSS rate of $89 \%$ compared to $37.5 \%$ in patients with simultaneous RVI (HR 3.54, $p=0.043$ ). In patients with SFI or combined PFI + SFI, each as a single variable, and for the combination with RVI, there was no statistically significant difference in terms of CSS between the respective groups ( $p=0.409$ and $p=0.202$ ). However, the 5-year CSS rate was reduced in groups with simultaneous RVI ( $82.6 \%$ vs. $60.6 \%$ for SFI and $85.7 \%$ vs. $57.5 \%$ for PFI+SFI, see Fig. 2f, g). In summary, these two unweighted prognostic risk stratification models could confirm the different prognosis in our inhomogeneous pT3a collective. The largest difference in terms of CSS was seen between patients with combined PFI+SFI with simultaneous RVI and patients with sole PFI (5-year CSS $57.5 \%$ vs $89.0 \%$, HR $4.01, p=0.029)$.

\section{Discussion}

The TNM classification has a great importance for risk stratification of RCC patients after tumor resection. Based on the 7th TNM edition from 2010, RCC with PFI, SFI or RVI alone or as a combination of these various options were summarized as pT3a tumors [4]. In the present study, we retrospectively examined the prognostic value of FI and also depending on the different fat compartments as well as RVI for medium-term CSS using 184 non-metastatic and metastatic RCC patients from an intern register. On univariate analysis for the total pT3a collective, the risk of tumordependent death was not significantly higher in patients with FI compared to sole RVI (HR 1.18, $p=0.591$ ). These discrepancies may result from the different cohort characteristics, since exclusively pT3a patients were included in our study and only 31 of these patients showed no FI but pure RVI. Previous work on the impact of FI on RCC patient outcome showed contradictory results, ranging from a negation, as with Siemer et al. and Gilbert et al., or the dependence on tumor diameter, as in Gofrit et al., to the reliable proof of an influence, reported by Brookman-May et al. [2, 5-7]. In our study, patients with FI and simultaneous RVI showed poorer CSS compared to patients with only FI $(p<0.01)$. This is consistent with other studies that have shown poorer CSS with a 2.6-fold increased risk of cancer-related death in patients with combined FI and RVI compared to cases with either of them alone $[8,9]$. At this point, the question arises whether the presence of a combined FI and RVI should be assessed differently in the TNM classification to better stratify patients with poor prognosis.

Taking into account the different fat compartments in the present work, there was no significant difference in the univariate analysis for the whole collective as well as the M0 subcollective for patients with PFI or SFI ( $p=0.079$ and 0.667). However, Thompson et al. described tumors with SFI as more aggressive compared to those with PFI with a 1.63-fold higher risk of tumor-dependent death [10]. In contrast, two recent studies found no influence of the location of FI on CSS in T3a RCC patients. [11, 12]. In our cohort, the survival of patients with combined PFI + SFI was significantly worse than in patients with only PFI or SFI $(p<0.01$ and $p=0.023)$ as well with no FI but sole RVI $(p=0.025)$. In the associated multivariate analyses, combined PFI+SFI could be confirmed as an independent negative prognostic factor for CSS in comparison to FI in only one of the two compartments (HR 1.82, $p=0.029$ ). However, combined PFI + SFI could not be identified as an independent negative prognostic factor for CSS compared to sole PFI $(p=0.172)$ or SFI $(p=0.062)$. In recent studies by Kume et al., Kresowik et al. and Bedke et al., a combined PFI+SFI represented an independent risk factor in multivariate analysis for a shorter CSS in RCC [13-15]. In contrast, Poon et al. and Margulis et al. could not confirm combined PFI+SFI compared to sole PFI as an independent predictor [11,12]. When comparing the described studies, it should be taken into account that both, the included patients, ranging from only localized RCC to metastatic diseases, and also the tumor classification differ significantly, since most studies used the TNM classification of 2002 $[5,7,16]$. Despite the intensive and careful documentation of the available data, the retrospective study design is a limiting factor and although the primary histopathologic analysis was made by uro-pathologists, no pathology re-review of the parrafin embedded tissue and the respective slides was performed. In the two unweighted models for prognostic risk stratification, we were able to show, on the one hand, the large difference in CSS in pT3a RCC patients ranging from 5-year CSS rates of 91.4\% in patients with FI and the parameters M0 L0 RVI0 to $44.9 \%$ in M0 L1 RVI1 patients (HR 4.89, $p<0.01$ ). On the other hand, we could demonstrate that CSS was significantly reduced in M0 patients of each affected fat compartment when RVI was present at the same time. The largest margin in terms 
of CSS was found between patients with combined PFI+SFI and additional RVI and patients with PFI alone without RVI (5-year CSS $57.5 \%$ vs. 89\%, HR 4.01, $p=0.029$ ). This observation is consistent with the studies by Guo et al. and Shah et al., who showed a significantly worse prognosis for patients with combined PFI+SFI and RVI [17, 18].

\section{Conclusions}

Taking into account the data obtained, the question arises whether the current TNM classification is sufficient to predict the risk of recurrence and survival of patients with pT3a RCC, or whether a division into a combined PFI + SFI compared to invasion in only one fat compartment and in particular the consideration of an additional RVI would be an important step towards better risk stratification. Furthermore, questions arise about the therapeutic consequences for patients with pT3a RCC with combined PFI + SFI with or without RVI. Some options could include a more frequent follow-up regime and review of adjuvant therapies to improve the expected poorer survival in these patients. In addition, this retrospective study highlights the importance of a careful, standard assessment of the type of FI. It is clear that larger studies will be required to further validate the prognostic value of combined PFI + SFI with simultaneous RVI in pT3a RCC.

Funding Open Access funding enabled and organized by Projekt DEAL. This research received no specific grant from any funding agency in the public, commercial, or not-for-profit sectors.

\section{Compliance with ethical standards}

Conflict of interest Dr. Bedke reports Consultancies and Speaker's Bureau: BMS; Eisai, EUSA, Ipsen, Novartis, MSD, Pfizer, Roche and study participation with institutional funding: Bayer, BMS; Eisai, Exelixis, Ipsen, Novartis, MSD, Pfizer, Roche. Dr. Stenzl reports Consultancies and Speaker's Bureau: Ipsen, Roche, Janssen, BMS, Alere, Stebabiotech, Synergo, Ferring, Astellas, Amgen, Sanofi Aventis, CureVac and study participation or research grants with institutional funding: Johnson \& Johnson, Roche, Cepheid, Amgen, Bayer, CureVac, GemeDx biotechnologies GmbH, Novartis, Karl Storz, immatics biotechnologies $\mathrm{GmbH}$. All other authors declare no conflict of interest.

Ethics approval The creation and retrospective review of our database was approved by the ethics committee of the University of Tuebingen, Germany, and, therefore, conforms to the ethical standards laid down in the 1964 Helsinki Declaration and its later amendments.

Informed consent Informed written consent was provided by each subject prior to surgical resection and the use of the tissue was approved by the ethics committee of the University of Tuebingen.
Open Access This article is licensed under a Creative Commons Attribution 4.0 International License, which permits use, sharing, adaptation, distribution and reproduction in any medium or format, as long as you give appropriate credit to the original author(s) and the source, provide a link to the Creative Commons licence, and indicate if changes were made. The images or other third party material in this article are included in the article's Creative Commons licence, unless indicated otherwise in a credit line to the material. If material is not included in the article's Creative Commons licence and your intended use is not permitted by statutory regulation or exceeds the permitted use, you will need to obtain permission directly from the copyright holder. To view a copy of this licence, visit http://creativecommons.org/licenses/by/4.0/.

\section{References}

1. Janzen NK, Kim HL, Figlin RA et al (2003) Surveillance after radical or partial nephrectomy for localized renal cell carcinoma and management of recurrent disease. Urol Clin 30(4):843-852

2. Brookman-May SD, May M, Wolff I et al (2015) Evaluation of the prognostic significance of perirenal fat invasion and tumor size in patients with pT1pT3a localized renal cell carcinoma in a comprehensive multicenter study of the CORONA project. Can we improve prognostic discrimination for patients with stage pT3a tumors? Eur Urol 67(5):943-951

3. Zhang Z, Yu C, Velet L et al (2016) The difference in prognosis between renal sinus fat and perinephric fat invasion for pT3a renal cell carcinoma: a meta-analysis. PLoS ONE 11(2):e0149420-e0149420

4. Edge SB, Compton CC (2010) The American Joint Committee on Cancer: the 7th edition of the AJCC cancer staging manual and the future of TNM. Ann Surg Oncol 17(6):1471-1474

5. Siemer S, Lehmann J, Loch A et al (2005) Current TNM classification of renal cell carcinoma evaluated: revising stage T3a. J Urol 173(1):33-37

6. Gofrit ON, Shapiro A, Pizov G et al (2007) Does stage T3a renal cell carcinoma embrace a homogeneous group of patients? J Urol 177(5):1682-1686

7. Gilbert SM, Murphy AM, Katz AE et al (2006) Reevaluation of TNM staging of renal cortical tumors: recurrence and survival for T1NOM0 and T3aNOM0 tumors are equivalent. Urology 68(2):287-291

8. da Costa WH, Moniz RR, da Cunha IW et al (2012) Impact of renal vein invasion and fat invasion in pT3a renal cell carcinoma. BJU Int 109(4):544-548

9. Baccos A, Brunocilla E, Schiavina R et al (2013) Differing risk of cancer death among patients with pathologic T3a renal cell carcinoma: identification of risk categories according to fat infiltration and renal vein thrombosis. Clin Genitourin Cancer 11(4):451-457

10. Thompson RH, Leibovich BC, Cheville JC et al (2005) Is renal sinus fat invasion the same as perinephric fat invasion for pT3a renal cell carcinoma? J Urol 174(4):1218-1221

11. Margulis V, Tamboli P, Matin SF et al (2007) Location of extrarenal tumor extension does not impact survival of patients with pT3a renal cell carcinoma. J Urol 178(5):1878-1882

12. Poon SA, Gonzalez JR, Benson MC et al (2009) Invasion of renal sinus fat is not an independent predictor of survival in pT3a renal cell carcinoma. BJU Int 103(12):1622-1625

13. Kume H, Homma Y, Shinohara N et al (2019) Perinephric invasion as a prognostic factor in non-metastatic renal cell carcinoma: analysis of a nation-wide registry program. Jpn J Clin Oncol 49(8):772-779

14. Kresowik TP, Johnson MT, Joudi FN (2010) Combined renal sinus fat and perinephric fat renal cell carcinoma invasion has a worse prognosis than either alone. J Urol 184(1):48-52

15. Bedke J, Buse S, Pritsch M et al (2009) Perinephric and renal sinus fat infiltration in pT3a renal cell carcinoma: possible prognostic differences. BJU Int 103(10):1349-1354 
16. Siddiqui SA, Frank I, Leibovich BC et al (2007) Impact of tumor size on the predictive ability of the pT3a primary tumor classification for renal cell carcinoma. J Urol 177(1):59-62

17. Guo S, Liu Z, Li X et al (2019) The prognostic value of the site of invasion in T3aNOM0 clear cell renal cell carcinoma. Urol Oncol 37(5):301.e11-301.e17

18. Shah PH, Lyon TD, Lohse CM et al (2019) Prognostic evaluation of perinephric fat, renal sinus fat, and renal vein invasion for patients with pathological stage T3a clear-cell renal cell carcinoma. BJU Int 123(2):270-276

Publisher's Note Springer Nature remains neutral with regard to jurisdictional claims in published maps and institutional affiliations. 